\title{
EVALUACIÓN DE LA RUGOSIDAD SUPERFICIAL Y EL COLOR DE LA RESINA BULK FILL CUANDO ES SOMETIDA A DISTIN- TOS PH SALIVALES
}

\author{
Evaluation of the surface rugosity and the color of the resin full in bulk when \\ subject to different ph salivals
}

\author{
Carolina Montserrat Cabezas Corral ${ }^{1}$, Alejandra Cabrera ${ }^{2}$, Ana del Carmen Armas Vega ${ }^{2}$ \\ ${ }^{1}$ Odontóloga, Facultad de Odontología, Universidad Central del Ecuador \\ ${ }^{2}$ Docente, , Facultad de Odontología, Universidad Central del Ecuador \\ DOI: https://doi.org/10.31984/oactiva.v6i3.579
}

\begin{abstract}
Resumen
Objetivo: Determinar el efecto en la rugosidad superficial y el color de una resina Bulk Fill, sometida a los diferentes $\mathrm{pH}$ salivales. Materiales y métodos: Se planteó un estudio de tipo experimental y comparativo utilizando 45 discos de $10 \mathrm{~mm}$ de diámetro y $1 \mathrm{~mm}$ de espesor, construidos a partir de una resina Bulk Fill, pulidos, tras revisión de su integridad, distribuidos de forma aleatoria en tres grupos $(n=15)$. Ejecutada la primera medición de rugosidad y color fueron sumergidos en frascos conteniendo saliva artificial cada uno con un $\mathrm{pH}$ diferente, 5, $6 \mathrm{y}$ 7 , durante 336 horas a una temperatura de $37^{\circ} \mathrm{C}$, para realizarse una nueva evaluación de rugosidad superficial y color, siguiendo a un nuevo contacto con las mismas sustancias hasta completar las 504 horas. Los datos obtenidos de las tres evaluaciones fueron recolectados en tablas de Excel, y analizados estadísticamente mediante pruebas de Kruskal Wallis, Anova y Tukey Resultados: Fue evidenciado una variación de la rugosidad de la resina estudiada proporcional al tiempo de contacto con las sustancias, de forma mas evidente cuando la sustancia alcanzó un pH 5 y 6. En cuanto al color, los datos obtenidos no permitieron ser analizados. Conclusión: Se evidencia un aumento en la rugosidad superficial directamente proporcional entre el tiempo de contacto y la acidez de la sustancia.
\end{abstract}

Palabras claves: $\mathrm{pH}$ Salival; rugosidad; color; composites.

\begin{abstract}
Objective: To determine the effect on both the surface roughness and color of a Bulk Fill resin, submitted to different salivary $p H$. Materials and methods: An experimental and comparative study was proposed using 45 discs from a Bulk Fill resin 10mm in diameter and $1 \mathrm{~mm}$ in thickness, built from a Bulk Fill resin and, after reviewing their integrity, they were polished and randomly distributed in three groups $(n=15)$. Once the first roughness and color measurement were carried out, they were immersed in flasks containing artificial saliva each one with a different pH: 5, 6 and 7 for 336 hours at a temperature of $37^{\circ} \mathrm{C}$, to then carry out a new evaluation of surface roughness and color, following a new contact with the same substances until 504 hours were completed. The data obtained from the three evaluations were collected in Excel, and statistically analyzed using the Kruskal Wallis, Anova and Tukey tests. Results: A variation in the roughness of the studied resin was evidenced, proportional to the time of contact with the substances, more clearly when the substance reached $\mathrm{pH} 5$ and 6 . The data obtained did not allow to analyze the color. Conclusion: There is evidence of an increase in surface roughness directly proportional between the contact time and the acidity of the substance.
\end{abstract}

Keywords: Salivary pH; rugosity; color; composites. 


\section{Introducción}

Las resinas compuestas, consideradas materiales de naturaleza polimérica, se han convertido en el material de elección en diferentes procedimientos operatorios, por su estética, propiedades físicas y mecánicas ${ }^{(1,2)}$, su evolución se ha presentado con mucha rapidez, encontrándose mejoras en su composición asociada a la incorporación de partículas de menor tamaño dentro de su composición inorgánica $^{(3,4)}$, desencadenando una nueva categoría de resinas denominadas Bulk Fill, con monómeros distintos de menor viscosidad, un mayor relleno inorgánico que resulta de la combinación de tricloruro de Iterbio y partículas de Zirconio, desencadenante de una manipulación amigable del material asociada a la colocación en bloque de la resina, en incrementos de hasta $4 \mathrm{~mm}$ de espesor. ${ }^{(5,6)}$

Evaluaciones mecánicas del material han comprobado las excelentes propiedades de las resinas tipo $\mathrm{Bulk}^{(7,8)}$; asociados a las modificaciones estructurales en su relleno ${ }^{(9)}$; sin embargo, ciertos hábitos alimenticios de los pacientes, asociados a cambios en el pH de la cavidad bucal, se muestran responsables de modificaciones en los materiales restauradores $^{(10)}$ muchos de ellos inevitables, aún frente a la composición y funciones de la saliva ${ }^{(11)}$, sobre todo cuando el desafío ácido sobre pasa los valores considerados críti$\cos ^{(12)}$. En este contexto, se hace necesario evaluar el impacto del tiempo de contacto de discos de resina Bulk Fill con sustancias de distintos $\mathrm{pH}$ salivales.

\section{Materiales y Métodos}

Se plantea un estudio del tipo experimental, comparativo con la aprobación del comité de investigación de la Facultad de Odontología de la Universidad Central del Ecuador.

La muestra del estudio estuvo constituida por 45 discos de resina Bulk Fill, siguiendo estudios previamente ejecutados ${ }^{(13)}$. Los 45 discos discos de resina con un diámetro de $10 \mathrm{~mm}$ y $1 \mathrm{~mm}$ de espesor fue ejecutada en un único incremento, empleando la lámpara de fotopolimerización (Woodpecker) como elemento de conversión del monómero a polímero; concluida su elaboración fueron sometidos a pulido mediante discos sof-lex en forma secuencial, durante 15 segundos en baja velocidad sin refrigeración, manteniendo los discos en ambiente húmedo durante todo el proceso.

A seguir los discos fueron mantenidos en recipientes oscuros con agua destilada y evaluados en su diámetro e integridad, descartándose aquellos que muestren signos de rayones, fracturas, porosidades o no se ajusten al diámetro establecido. Una vez, divididos aleatoriamente fueron identificados y sometidos a la primera evaluación de su rugosidad superficial mediante rugosímetro digital (Mitutoyo) y colorímetro (VITA), los valores obtenidos adecuadamente almacenados en tablas previamente elaboradas. Las muestras divididas en 3 grupos (n:15), se almacenaron a una temperatura de $37^{\circ}{ }^{(8)}$ en incubadora(14) "Incucell", en recipientes conteniendo $20 \mathrm{ml}$ de saliva artificial (Salivsol) modificada químicamente y valorada mediante un pH metro, hasta obtener un pH 5, 6 y 7; con cambios de la sustancia a cada 24 horas.

La evaluación de rugosimetría fue ejecutada por 3 ocasiones sobre cada disco de resina mediante un rugosímetro digital (TESTER SRT-6200). El primer registro fue realizado posterior al pulido de los discos de resina, el segundo registro se realizó a las 336 horas ${ }^{(8,16)}$ de estar sumergidos los discos en saliva artificial a los diferentes pH y a las 504 horas.

La evaluación del color fue registrada mediante el colorímetro digital (Tooth Color Comparator), colocando cada muestra a $2 \mathrm{~mm}$ de distancia de la punta medidora del colorímetro, evitando la existencia de luz artificial, que pueda modificar el color de las muestras; los valores obtenidos fueron comparados con los colores de la escala VITA, registrando los valores obtenidos en los tres periodos de evaluación.

Los datos recolectados tras la medición de la rugosidad y el color fueron ingresados en tablas de Excel, y posteriormente analizados estadísticamente con pruebas de Kruskal Wallis, Anova y Tukey.

\section{Resultados}

Las evaluaciones en los tres tiempos considerando el $\mathrm{pH}$ de la sustancia en contacto, determino cambios en la rugosidad directamente proporcionales a la acidez de la sustancia en contacto, que incremento con el tiempo de contacto con la sustancia, con una media de $0,479 \mu \mathrm{m}$ a los 21 días, con un pH 5 de la sustancia, similares resultados se encontraron con pH 6 a los 21 días una media de $0,436 \mu \mathrm{m}$ y con un $\mathrm{pH} 7$ a los 21 días con uba nedua de $0,394 \mu \mathrm{m}$.

En referencia a la coloración de la resina según la escala VITA los cambios fueron evidentes pero mostraron dificultad en cuanto a la forma de registrarlos por las limitaciones propias del sistema de medición empleado. Con respecto a los datos de rugosidad en los tres tiempos, los datos fueron analizados en el programa SPSS, ejecutándose la prueba de normalidad con Shapiro Wilk que permitió determinar que se trata de datos de distribución normal, por lo cual 
fueron ejecutadas pruebas paramétricas de T Student, Anova y Tukey, que evidenciaron una evidente relación entre los distintos $\mathrm{pH}$ y la rugosidad superficial observada en la resina Bulk Fill en relación directa con los tiempos de almacenaje $(\mathrm{p}<0,05)$.

\section{Discusión}

Se evidenció una dirección directamente proporcional entre tiempo de contacto con la sustancia y la acidez de la sustancia, con mayores cambios en la rugosidad coincidiendo con estudios similares ${ }^{(8)}$ con cambios notorios en la microdureza de estos materiales ${ }^{(14)}$, asociados a la composición de la estructura química de las resinas y al contenido de las partículas de relleno ${ }^{(9)}$ asociado a la capacidad de absorción y solubilidad de los líquidos, que puede llegar a adherirse el fluido a la interface entre la partícula de relleno inorgánico y la matriz polimérica, provocando la degradación de la resina ${ }^{(8,13)}$, sin embargo, resultados diferentes fueron encontrados al evaluar la rugosidad superficial de una resina compuesta sumergida en enjuagues bucales con y sin alcohol ${ }^{(13)}$ que estarían asociados a la capacidad hidrofóbica de los materiales resinosos, los cambios drásticos en el pH salival que pueden recibir las diferentes resinas en el ambiente bucal son factores determinantes para que exista un desgaste y una variación en la rugosidad con el paso del tiempo, dicha variación puede ser significativa o insignificante dentro del contexto de los estudios realizados ${ }^{(15)}$.

La determinación de las alteraciones en el color de los materiales también fue uno de los objetivos de este estudio; sin embargo, la metodología empleada para evaluación, no permitió visualizar modificaciones en ellas. Estudios previos determinaron alteraciones de las resinas compuestas en cuanto a la translucidez y aspereza cuando las resinas compuestas fueron sometidas a diferentes bebidas de forma cíclica, con evidente disminución de translucidez ${ }^{(9)}$, en el estudio los rangos de cambio de color fueron mínimos y los datos no permitieron la ejecución de un análisis estadístico, lo que estaría asociado al colorímetro, como instrumento empleado. Resulta evidente el hecho de que la pigmentación, se produce de acuerdo al tipo de sustancia empleada ${ }^{(15,20)}$ entendiendo que el color de un material no puede ser percibido únicamente por color, elementos como translucidez y opalescencia deben ser considerados.

Una de las principales limitaciones del estudio constituye el hecho de haber sido planteado como un estudio de tipo experimental, donde si bien a través de saliva artificial se intenta simular las condiciones bucales, dentro de la cavidad bucal, existe un sinnúmero de interacciones que influ- yen sobre el comportamiento de un material ${ }^{(8,23)}$, de ahí la necesidad de ejecutar nuevas investigaciones considerando otros elementos de análisis, microdureza, resistencia masticatoria conjugada con otros materiales y condiciones como espesor, tiempo de foto curado y longevidad; considerando sistemas de medición del color, específicos.

Como clínicos, resulta importante considerar la responsabilidad de guiar y asesorar al paciente que recibe resinas compuestas, como material restaurador, en cuanto al consumo de bebidas con los diferentes $\mathrm{pH}$ salivales, considerando las modificaciones en la estructura de estos materiales y los elementos que los pueden provocar.

\section{Conclusiones}

Al evaluar los tres grupos de discos de resina Bulk Fill en los distintos periodos de tiempo, el grupo que más cambios en la rugosidad superficial tuvo, fue el que estaba sumergido en $\mathrm{pH} 5$ a los 21 días, y en el color la significancia fue mínima.

\section{Referencias}

1. Pérez A, Fereira J, Espina Á, Ortega A. Cambios estructurales de las resinas compuestas sometidas a la acción de altas temperaturas. Ciencia odontológica. ; 14(1): p. 25-34.

2. Zhou W, Liu S, Zhou X, Hannig M, Rupf S, Feng J, et al. Modifying Adhesive Materials to Improve the Longevity of Resinous Restorations. Int J Mol Sci. 2019 Feb; 20(3): p. 35-

3. Molina G., Palma S. Nanotecnologia en Odontologia: Aspectos generales y posibles aplicaciones. Rev Methodo. 2018;3(3):59-66.

4. Dursun E, Fron-Chabouis H, Attal J, Raski A. Bisphenol A Release: Survey of the Composition of Dental Composite Resins. Open Dent J. 2016; 10(1): p. 446453.

5. Thakarshibhai R, Batra R, Kapoor S. Comparative Evaluation of Postoperative Sensitivity in Bulk Fill Restoratives: A Randomized Controlled Trial. J Int Soc Prev Community Dent. 2018 Nov-Dec; 8(6): p. 534-539.

6. Salagalla U, Mandava J, Ravi C, Nunna V. Effect of intratooth location and thermomechanical cycling on microtensile bond strength of bulk-fill composite resin. 2018; 21(6): p. 657-661. 
7. Moradas M, Álvarez B. Dinámica de polimerización enfocada a reducir o prevenir el estrés de contracción de las resinas compuestas actuales. Revisión bibliográfica. Av Odontoestomatol. 2017 nov./dic; 33(6): p. 261-272.

8. Alshali R, Salim N, Satterthwaite J, Silikas N. Longterm sorption and solubility of bulk-fill and conventional resin-composites in water and artificial saliva. $\mathrm{J}$ Dent. 2015 Dec; 43(12): p. 1511-8.

9. Yu H, Cheng S, Jiang N, Cheng H. Effects of cyclic staining on the color, translucency, surface roughness, and substance loss of contemporary adhesive resin cements. J Prosthet Dent. 2018; 120(3): p. 462-469.

10. Schroeder T, da Silva P, Basso G, Franco M, Maske T, Cenci M. Factors affecting the color stability and staining of esthetic restorations. Odontology. 2019 marzo; 1(1): p. 1-6.

11. Saenz, M. F; Madrigal, D. Capacidad Buffer de la saliva y su relación con la prevalencia de con la ingesta de bebidas comerciales. Odontología Vital. 2019 31:59-66.

12. Roa I, del Sol M. Obesity, salivary glands and oral pathology. Colomb Med (Cali). 2018; 49(4): 80-87.

13. Casanova O, Taboada A, Flores C, et al. Efecto de tres enjuagues bucales en la degradación superficial de resinas compuestas: estudio in vitro. Rev Odontopediatr Latinoam. 2018;8(2):141-153.

14. Guler S, Unal M. The Evaluation of Color and Surface Roughness Changes in Resin based Restorative Materials with Different Contents After Waiting in Various Liquids: An SEM and AFM study. Microsc Res Tech. 2018; 81(12): p. 1422-1433.

15. Arcos Tomal LC, Montaño Taté VA, Armas AC. (2019). Estabilidad en cuanto a color y peso, de resinas compuestas tipo flow tras contacto con bebidas gaseosas: estudio in vitro. Odontología Vital, (30), 59-64. Retrieved December 11, 2020.

16. Carrillo M, Ugrate D, Benitez A, Filho P. Evaluación in vitro de la rugosidad superficial y la alteración de color de dos tipos de ionómeros de vidrio, luego de ser sometidos a diferentes bebidas. Revista de odontopediatría latinoamericana. 2017; 7(2): p. 35-42.

17. Peñafiel Rodríguez, M. V., Quisiguiña Guevara, S. M., Alban Hurtado, C. A., \& Robalino Campos, H. R. (2019). Comparación de la resistencia a la fuerza de compresión de las resinas híbrida, nanohíbrida y bulk fill. RECIMUNDO, 3(3), 585-595.

18. Haugen, H.J.; Marovic, D.; Par, M.; Khai Le Thieu, M.; Reseland, J.E.; Johnsen, G.F. Bulk Fill Composites Have Similar Performance to Conventional Dental Composites. Int. J. Mol. Sci. 2020, 21, 5136.

19. Besegato J, Jussiani E, Andrello A, Fernandes R, Salomão F, Santana B, et al. Effect of light-curing protocols on the mechanical behavior of bulk-fill resin composites. Journal of the Mechanical Behavior of Biomedical Materials. 2019; 90(1): p. 381-387.

20. Zini C, González, M; Martínez, S. la saliva: una mirada hacia el diagnóstico. 2016. RAAO, 55 (2): 40- 43.

21. Marsh P, Do T, Beighton D, Devine D. Influence of saliva on the oral microbiota. Periodontol 2000. 2016; 70(1): p. $80-92$.

22. Obreque E, Espínola V, López R. Wine pH Prevails over Buffering Capacity of Human Saliva. J. Agric. Food Chem. 2016; 64(43): p. 8154-8159.

23. Gésime J.M, Merino R.L, Briceño E.N Influencia del PH en las relaciones microbianas de la cavidad bucal 2018.

Recibido: 01 de marzo de 2021

Aceptado: 01 de mayo de 2021 